\title{
The Influence of Sheath Solvent Compositions on the Diameters of Electrospun PCL/PTMC Nanofibers
}

\section{Zhao-Bin ZHENG ${ }^{1, a}$, Chen $\mathrm{Li}^{2}$, Yang-Chao DENG ${ }^{1}$, Yang-Lu QU ${ }^{1}$, Di WU ${ }^{1}$ and Deng-Guang $Y \mathrm{U}^{1, \mathrm{~b}, *}$}

\author{
${ }^{1}$ School of Material Science and Engineering, University of Shanghai for Science and \\ Technology, Shanghai 200093, P.R. China \\ ${ }^{2}$ Institute of Biotechnology, Shanxi University, Taiyuan030006, P.R. China \\ a turbozb@126.com; b ydg017@usst.edu.cn \\ ${ }^{*}$ Corresponding author
}

Keywords: Coaxial electrospinning, Nanofiber, Nanofabrication, Composite, Shell fluid

\begin{abstract}
The modified coaxial electrospinning, in which only organic solvents or their mixtures are utilized as sheath working fluids, is a useful way for manipulating nanofibers' diameters. In this study, with a co-dissolving solution consisting of poly( $\varepsilon$-caprolactone) (PCL) and poly(trimethylene carbonate) (PTMC) at a concentration of $12 \mathrm{wt} \%$ in a mixture of dichloromethane (DCM)/N,N-dimethylformamide (DMF) as the core fluid, a series of solvent mixtures with a varied compositions of them were exploited as the sheath fluids to control the sizes of electrospun PCL/PTMC nanofibers. The scanning electron microscopic results demonstrated that the nanofibers' diameter (D) had a fine relationship with the DMF volume ratio $\left(V_{r}\right)$ in the sheath fluids as $D=162 V_{r}-0.61\left(R^{2}=0.9922\right)$. This job opens a new way for conducting the modified coaxial processes and for adjusting the sizes of fibers intentionally.
\end{abstract}

\section{Introduction}

Electrospinning, together with electrospraying and e-jetting printing, are commonly termed as electrohydrodynamic atomization (EHDA) techniques, which are simple single-step "top-down" processes for nano fabrications [1-7]. Different with traditional methods, electro-static energy is directly exploited to remove organic solvents from the fluids for generating solid products during the EHDA processes [8-13]. These advanced processes are popular in many fields such as pharmaceutics, ceramics, energy and environments [14-19].

However, the intentioal manipulation of nanofibers' diameters is still one of the most intractable problems in electrospinning. Although many efforts have been paid to this goal, the results are far from being satisfied [20]. Even the aim of obtaining thinning nanofibers is often compromised by the detriment of nanofiber quality in a single fluid electrospinning process. New strategy that can produce high quality nanofibers stably and controllably is highly desired.

Coaxial electrospinning was initially applied in controlling secondary structures of nanofibers, later was expaneded to the applications of encapsulating drugs or biological agents into the polymeric nanofibers $[21,22]$. During the coaxial process the sheath solution acts as a guide and surrounds the core material, and the viscosity of the sheath solution is required to overcome interfacial tension between the two solutions so enabling the formation of a compound Taylor cone and a constant jet. However, this traditional idea was broken by $\mathrm{Yu}$. A modified process based on coaxial and triaxial electrospinning was reported, where only 
solvent as used as a sheath fluid [23-26]. The new process has been demonstrated to be useful for stabilzing the electrospinning process and for manipulating the nanofibers' diameters.

\section{Materials and Methods}

\section{Materials}

Poly( $\varepsilon$-caprolactone) (PCL, $\left.\mathrm{M}_{\mathrm{w}} \sim 80,000\right)$ and poly(trimethylene carbonate) (PTMC, $\mathrm{M}_{\mathrm{w}} \sim$ 100,000) were provided by Minghe Functional Polymer Co., Ltd. (Qingdao, China). Dichloromethane (DCM) and N,N-dimethylformamide (DMF) were purchased from Sinopharm Chemical Reagent Co., Ltd. (Shanghai, China).

\section{Preparations}

A fixed blend ratio of PCL and PTMC ( 6:4, w/w) were dissolved in a mixture of DCM/DMF with a volume ratio of $70: 30(\mathrm{v}: \mathrm{v})$ to prepare the core fluid at a concentration of $12 \mathrm{wt} \%$. A series of mixture consisting of DCM and DMF with varied volume ratio were exploited as the sheath working fluids.

A ZGF60 kV/2 mA power supply (Wuhan Huatian Corp., Wuhan, China), two KDS100 syringe pumps (Cole-Parmer ${ }^{\circledR}$, Vernon Hills, USA) and a cardboard wrapped with aluminum foil were utilized to set up the in-house electrospinning system. After some pre-experiments, the applied voltage and the spinneret-to-collector distance were fixed at $16 \mathrm{kV}$ and $20 \mathrm{~cm}$, respectively. The feeding rate of the sheath and core fluids were adjusted at 0.2 and $0.8 \mathrm{~mL} / \mathrm{h}$, respectively. Nanofibers prepared from a DCM/DMF volume ratio of 100/0, 80/20, 60/40, 40/60, 20/80 were denoted as F1, F2, F3, F4 and F5, respectively.

\section{Characterizations}

The morphology of the fibers was evaluated using a scanning electron microscope (SEM; FEI Quanta 200 FEG ESEM instrument). Each specimen was fixed with conductive double-sided carbon adhesive tape and were gold sputter-coated under a nitrogen atmosphere to render them electronically conductive. The average fiber diameter was determined by measuring their sizes in SEM images at more than 100 different places using the ImageJ software (National Institutes of Health, Bethesda, MD, USA).

\section{Results and Discussion}

\section{The Modified Coaxial Processes}

Shown in Fig. 1a is a schematic diagram of the coaxial system, which was composed of the typical four components, i.e. the two syringe pumps, the spinneret, the fiber collector and the high voltage power supply. The key component is the spinneret, which determines what types of electrospinning processes are carried out (Fig. 1b). 


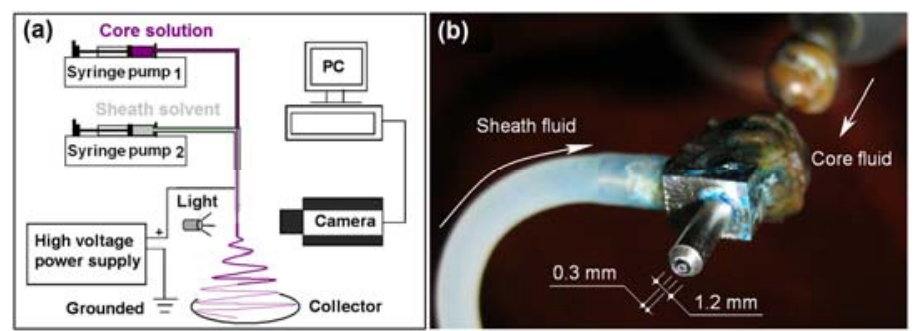

Fig 1. A schematic diagram of the modified coaxial electrospinning (a), and its key component, the concentric spinneret (b).

A digital picture of the electrospinning system is shown in Fig. 2a. The connections of the spinneret with working fluids and power supply are shown in Fig. 2b, in which the core fluid syringe was directly connected with the spinneret and the sheath fluid syringe was connected with the spinneret through a highly elastic silicon tubing. The high voltage was applied on the two fluids through an alligator clip.

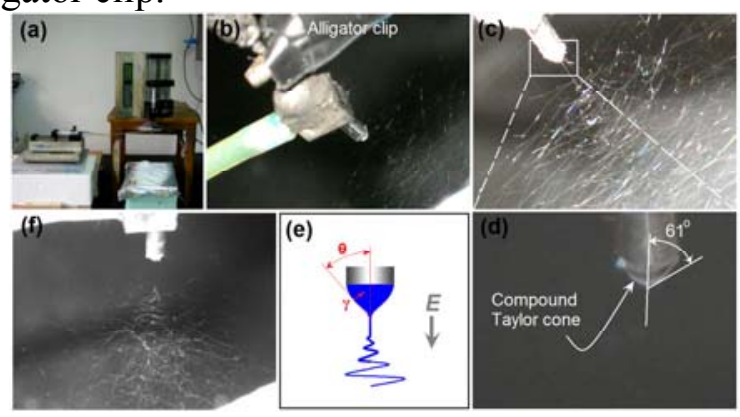

Fig 2. Implementation of the modified coaxial electrospinning. (a) The electrospinning system; (b) The connections of the spinneret; (c) A digital image for fabricating nanofibers F2; (d) The compound Taylor cone for preparing fibers F2; (e) A schematic diagram showing the fluid jet emitted half-vertical angle; (f) A digital image for fabricating nanofibers F5.

An image of the real electrospinning process for preparing nanofibers F2 is shown in Fig. 2c. It was a typical process, starting from a Taylor cone, through which a straight fluid jet was emitted, and followed by a continuously enlarged bending and hipping loops. Its compound Taylor-cone was exhibited in Fig .2d, in which an obvious image of half an core-shell structure. The working semi-vertical angle was $61^{\circ}$. It is a common sense that the semi-vertical angle $\theta$ reflects a balance between the the electrical field $(E)$ and the surface tension of working fluid $(\gamma)$ in Fig. 2e, and its value should be at the range of $32^{\circ}$ to $46^{\circ}$ for a stable and robust electrospinning process [27]. In the present modified coaxial process, the value of $\theta$ was out of the range a lot, but the coaxial process was still stable and continuously. However, when the sheath mixture contained too much DMF, the coaxial process lost its stability to some extents. A typical enlarged image of the process for fabricating fibers F5 is exhibited Fig. $2 \mathrm{f}$. It is obvious that the bending loops couldn't be fully expand.This should have a close relationship with the physical property of solvent DMF, which has a high boiling point of $153{ }^{\circ} \mathrm{C}$. 


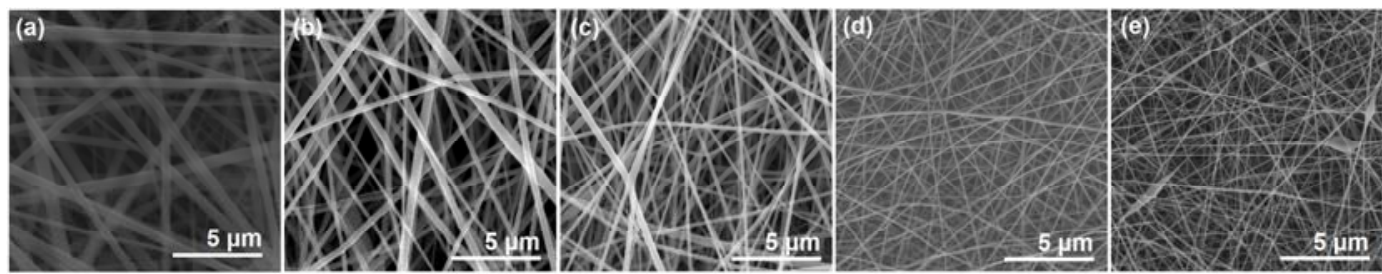

Fig 3. The SEM images of the prepared nanofibers: (a) F1; (b) F2; (c) F3; (d) F4; (e) F5.

\section{Morphology}

The SEM images of the five kinds of nanofibers are exhibited in Fig. 3. All the nanofibers had the linear morphology without any spindles-on-a-string phenomena except nanofibers F5. Although nanofibers F5 (Fig. 3e) showed a smaller diameter than the others (Fig. 3a to 3d), these fibers had many spindles-on-a-string morphology. It should be the excessive DMF in the sheath mixture that resulted in these morphologies.

\section{The Influence of Solvent-to-solvent Ratio in the Shell Fluid on the Electrospun Nanofibers}

The average diameter and distribution of the prepared five types of nanofibers are shown in Fig. 4. As the volume ratio of DMF in the sheath mixture increased from $0 \%$ to $20 \%, 40 \%, 60 \%$ and $80 \%$, their avarage diameters gradually decreased from $610 \pm 150 \mathrm{~nm}$, to $460 \pm 110 \mathrm{~nm}, 270 \pm$ $80 \mathrm{~nm}, 220 \pm 50 \mathrm{~nm}$, and $180 \pm 50 \mathrm{~nm}$ for nanofiber F1 to F5, respectively. The more DMF in the sheath fluid, the more difficult it was exhausted during the electrospinning. Thus in turn, the longer time period the fluid jets were subjected to the electrical force drawing and the nanofibers with smaller diameters were generated.

To further disclose the above-mentioned trend and find the inner relationship between the nanoifbers' size and the sheath solvent compositions, both linear equation and exponential equation were exploited to fit the statistical data. The results were shown in Fig. 5a (linear equation) and $5 \mathrm{~b}$ (exponential equation). The linear equation is $\mathrm{D}=557-530 \mathrm{~V}_{\mathrm{r}}$ with a correlation coefficient of $\mathrm{R}^{2}=0.9023$, reflecting a poor linear relationship between them. However, the regressed exponential equation $\mathrm{D}=162 \mathrm{~V}_{\mathrm{r}}^{-0.61}$ gives a correlation coefficient value of $\mathrm{R}^{2}=0.9922$, suggesting a fine relationship. Thus, the exponential equation was better than the linear one in reflecting the influence of sheath solvent compositions on the nanofibers' diameters, and it could be effectively utilized to accurately predict the diameter of electrospun nanofibers.

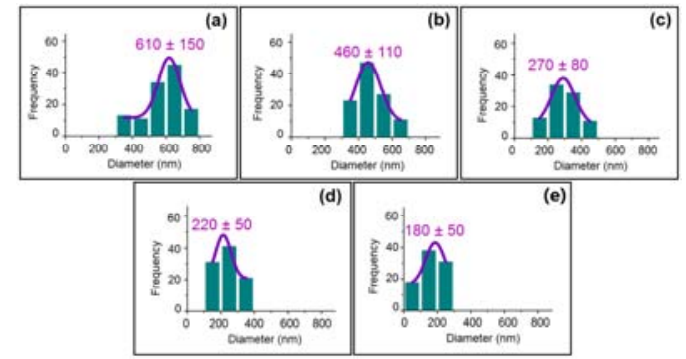

Fig. 4 The average diameter and distribution of the prepared nanofibers: (a) F1; (b) F2; (c) F3; (d) F4; (e) F5. 


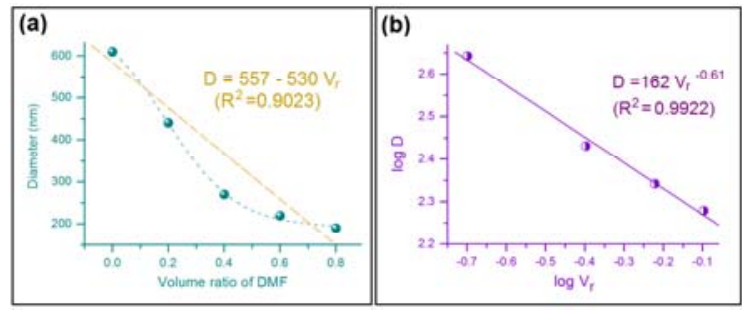

Fig. 5 Different equations were exploited to fit the statistical data about diameters: (a) linear equation; (b) exponential equation.

\section{Conclusions}

A series of modified coaxial processes have been successfully carried out for preparing PCL/PTMC composite nanofibers. The solvent mixtures consiting of DMF and DCM and with a varied of compositions were exploited as the sheath fluids to control the sizes of electrospun nanoproducts. The SEM results demonstrated that the nanofibers' diameter (D) had a quantative relationship with the DMF volume ratio $\left(V_{r}\right)$ in the sheath fluids as $D=162 V_{r}{ }^{-0.61}$ $\left(\mathrm{R}^{2}=0.9922\right)$. It can be concluded that the present job paved a new way for implementing the modified coaxial electrospinning processes and thediameters of resulted nanofibers.

\section{Acknowledgements}

The financial supports were from the following projects: the College Student Innovation Project of USST (Nos. XJ2017234 and SH2017176), the Natural Science Foundation of Shanxi Province (No. 2015021047) and the Science \& Technology Innovation Project in High School of Shanxi Province (No. 2016116).

\section{References}

[1] Z.P. Liu, L. Cui, D.G. Yu, Z.X. Zhao and L. Chen. Electrosprayed core-shell solid dispersions of acyclovir fabricated using an epoxy-coated concentric spray head. Int. J. Nanomed., 9 (2014) 1967-19775.

[2] Y.H. Wu, D.G. Yu, J.J. Li, Q. Wang, H.P. Li and X.Y. Li, Medicated multiple-component polymeric nanocomposites fabricated using electrospraying, Polym. Polym. Compos., 25 (2017) 57-62.

[3] J. Yan, Y.H. Wu, D.G. Yu, G.R. Williams, S.M. Huang, W. Tao and J.Y. Sun, Electrospun acid-base pair solid dispersions of quercetin, RSC Adv., 4 (2014), 58265-58271.

[4] M. Jin, D.G. Yu, C.F.G.C. Geraldes, G.R. Williams, S.W.Annie Bligh, Theranostic fibers for simultaneous imaging and drug delivery, Mol. Pharm., 13 (2016) 2457-2465.

[5] D.G. Yu, K. White, N. Chatterton, Y. Li, L. Li, X. Wang, Structural lipid nanoparticles self-assembled from electrospun core-shell polymeric nanocomposites, RSC Adv., 5 (2015) 9462-9466. 
[6] D.G. Yu, X.X. Shen, C. Brandford-White, K. White, L.M. Zhu, S.W.A. Bligh, Oral fast-dissolving drug delivery membranes prepared from electrospun polyvinylpyrrolidone ultrafine fibers, Nanotechnology, 20 (2009) 055104.

[7] J. Xie, H. Mao, D.G. Yu, G.R. Williams and M. Jin, Highly stable coated polyvinylpyrrolidone nanofibers prepared using modified coaxial electrospinning, Fiber. Polym., 15 (2014) 78-83.

[8] J. Yan, K. White, D.G. Yu and X.Y. Zhao, Sustained release multiple-component cellulose acetate nanofibers fabricated using a modified coaxial electrospinning process, J. Mater. Sci., 49 (2014) 538-547.

[9] C. Li, Z.H. Wang and D.G. Yu, Higher quality quercetin sustained release ethyl cellulose nanofibers fabricated using a spinneret with a Teflon nozzle, Colloid. Surface. B, 114 (2014): 404-409.

[10]W. Qian, D.G. Yu, Y. Li, Y.Z. Liao, X. Wang and L. Wang, Dual drug release electrospun core-shell nanofibers with tunable dose in the second phase, Int. J. Mol. Sci., 15 (2014) 774-786.

[11]D.G. Yu, Y. Xu, Z. Li, L.P. Du, B.G. Zhao and X. Wang. Coaxial electrospinning with mixed solvents: From flat to round Eudragit L100 nanofibers for better colon-targeted sustained drug release profiles, J. Nanomater., 2014 (2014) 967295.

[12]L. Cui, Z.P. Liu, D.G. Yu, S.P. Zhang, S.W.A. Bligh, N. Zhao, Electrosprayed core-shell nanopartciles of PVP and shellac for furnishing biphasic controlled release of ferulic acid. Colloid. Polym. Sci., 292 (2014) 2089-2096.

[13]X.Y. Li, Y.C. Li, D.G. Yu, Y.Z. Liao, X. Wang, Fast disintegrating quercetin-loaded drug delivery systems fabricated using coaxial electrospinning, Int. J. Mol. Sci., 14 (2013) 21647-21659.

[14]C. Li, D.G. Yu, G.R. Williams and Z.H. Wang. Fast-dissolving core-shell composite microparticles of quercetin fabricated using a coaxial electrospray process, PLOS One, 9 (2014) e92106.

[15]Y.H. WU, C. Yang, X.Y. Li, J.Y. Zhu, D.G. Yu, Medicated nanofibers fabricated using $\mathrm{NaCl}$ solutions as shell fluids in a modified coaxial electrospinning, J. Nanomater., 2016 (2016) 8970213.

[16] G.Z. Yang, H.P. Li, J.H. Yang, J. Wan and D.G. Yu, Influence of working temperature on the formation of electrospun polymer nanofibers. Nanoscale Res. Lett., 12 (2017) 1-10.

[17]F. Xu, Y. Xu and D.G. Yu, Zero-order controlled release nanofibers fabricated using coaxial electrospinning with polymer dilute solution as a sheath fluid, J. University of Shanghai for Science and Technology, 37 (2015) 165-168.

[18]D.G. Yu, J.J. Li, M. Zhang, G.R. Williams, High-quality Janus nanofibers prepared using three-fluid electrospinning, Chem. Commun., 53 (2017) 4542-4545. 
[19] D.G. Yu, C. Yang, M. Jin, G.R. Williams, H. Zou, X. Wang, S.W.A. Bligh, Medicated Janus fibers fabricated using a Teflon-coated side-by-side spinneret, Colloid. Surface. B, 138 (2016) 110-116.

[20]H.F. Wen, C. Yang, D.G. Yu, X.Y. Li and D.F. Zhang, Electrospun zein nanoribbons for treatment of lead-contained wastewater, Chem. Eng. J. 290 (2016), 263-272.

[21]C. Li, Z.H. Wang, D.G. Yu and G.R. Williams, Tunable biphasic drug release from ethyl cellulose nanofibers fabricated using a modified coaxial electrospinning process, Nanoscale Res. Lett., 9 (2014) 258.

[22]C. Yang, D.G. Yu, D. Pan, X.K. Liu, X. Wang, S.W. A. Bligh and G. R. Williams, Electrospun $\mathrm{pH}$-sensitive core-shell polymer nanocomposites fabricated using a tri-axial processes, Acta Biomater. 35 (2016) 77-86.

[23]D.G. Yu, X.Y. Li, X. Wang, J.H. Yang, S.W.A. Bligh, G.R. Williams, Nanofibers fabricated using triaxial electrospinning as zero order drug delivery systems, ACS Appl. Mater. Interfaces 7 (2015) 18891-18897.

[24]C. Sealy, New spin on drug-loaded composite fibers, Materials Today, 19 (2016) 374-374.

[25]D.G. Yu, X.Y. Li, W. Chian, Y. Li and X. Wang, Influence of sheath solvents on the quality of ethyl cellulose nanofibers from a modified coaxial electrospinning, Biomed. Mater. Eng., 24 (2014) 695-701.

[26]G.Z. Yang, J.J. Li, D.G. Yu, M.F. He, J.H. Yang and G.R. Williams, Nanosized sustained-release drug depots fabricated using modified tri-axial electrospinning, Acta Biomater., 53 (2017) 233-241.

[27]D.G. Yu, G.R. Williams, X. Wang, X.K. Liu, H.L. Li, S.W.A. Bligh, Dual drug release nanocomposites prepared using a combination of electrospraying and electrospinning, RSC Adv., 3 (2013) 4652-4658. 